\title{
Efeitos de Rações com Níveis Crescentes de Cana-de-Açúcar em Substituição à Silagem de Milho sobre a População de Protozoários Ciliados no Rúmen de Ovinos
}

\author{
Maria Helena Tieghi Franzolin', Carlos de Sousa Lucci², Raul Franzolin ${ }^{3}$
}

\begin{abstract}
RESUMO - Oito ovinos machos com fístulas ruminais foram delineados em dois quadrados latinos, em quatro períodos de 35 dias cada e quatro tratamentos, compreendendo níveis crescentes de cana-de-açúcar (CA) em substituição à silagem de milho (SM): A) 100\% SM; B) 67\% SM e 33\% CA; C) 33\% SM e 67\% CA; e D 100\% CA, para avaliação da população de protozoários ciliados no rúmen. As concentrações totais de protozoários ciliados no rúmen e de Entodinium diminuíram e os valores de pH do conteúdo ruminal aumentaram linearmente com o incremento das quantidades de cana-de-açúcar na ração. Não houve diferenças entre tratamentos para o volume ruminal e a taxa de passagem da fase líquida do rúmen.
\end{abstract}

Palavras-chave: cana-de-açúcar, ovinos, protozoários do rúmen

\section{Effects of Diets with Increasing Levels of Sugar Cane in Substitution of Corn Silage on Rumen Population Ciliate Protozoa in Sheep}

\begin{abstract}
Eight rumen fistulated rams were delineated in two Latin Squares in four periods of 35 days each and four treatments consisted of increasing levels of sugar cane (SC) in substitution to the corn silage (CS): A) $100 \%$ CS, B) $67 \%$ CS and $33 \%$ SC, C) $33 \%$ CS and $67 \%$ SC and D) $100 \%$ SC, to evaluate the population of ciliate protozoa in the rumen. The total concentrations of rumen ciliate protozoa and of Entodinium decreased and the values of $\mathrm{pH}$ of the ruminal content increased linearly as dietary sugar cane levels increased. No differences between treatments were observed for the rumen volume and the rumen liquid turnover rate.
\end{abstract}

Key Words: sugar cane, sheep, rumen protozoa

\section{Introdução}

Os ciliados do rúmen participam com mais de $40 \%$ do nitrogênio microbiano total do conteúdo do órgão e mais de $60 \%$ dos produtos de fermentação microbiana (HUNGATE, 1966). A quantidade e diversidade são influenciadas pelas dietas ingeridas, pelo $\mathrm{pH}$ do conteúdo ruminal e pelas relações que estabelecem entre si e com a população bacteriana (MOURA MARINHO, 1982). LENG (1982) observou que 65\% dos prozotoários morrem e são degradados no rúmen.

Segundo CHURCH (1974), o amido é fermentado pelos protozoários ciliados a ácidos graxos voláteis. Em ruminantes submetidos à dietas ricas em açúcares, os protozoários holotricos digerem rapidamente os carboidratos solúveis, convertendo-os em amido. Aproximadamente 30\% dos açúcares ingeridos pelos animais seriam convertidos em amilopectina pelos protozoários (COLEMAN, 1979).

De acordo com DEHORITY e PURSER (1970), a diminuição na ingestão dos alimentos tende elevar a população dos protozoários. CHRISTIANSEN et al. (1964) explicaram que a ingestão reduzida de alimentos ocasiona menor taxa de passagem dos nutrientes pelo rúmen, favorecendo, assim, o estabelecimento e crescimento dos prozotoários.

Vários trabalhos foram desenvolvidos sobre os efeitos da administração da cana-de-açúcar (CA) para ruminantes. BIRD et al. (1979) encontraram população de protozoários no rúmen de ovino na ordem de 5,0 x $10^{5}$ por mL de líquido ruminal com os entodínios, representando $96 \%$ do total. VALDEZ et al. (1977) observaram números elevados de protozoários ruminais, com predominância de Isotricha intestinalis e Dasytrica ruminatium, porém as espécies de Entodium estavam presentes, embora em número menor. MINOR et al. (1977), utilizando bovinos alimentados com cana-de-açúcar ad libitum e uréia, durante dois meses, observaram concentração de $2,48 \times 10^{5}$ de holotricos e $3,0 \times 10^{5}$ de entodínios por $\mathrm{mL}$ de líquido ruminal.

Em dietas com cana-de-açúcar, segundo LENG e

\footnotetext{
1 Zootecnista, Doutora em Nutrição e Produção Animal Animal pela FMVZ/UNESP - Botucatu, SP.

2 Professor Titular da Faculdade de Medicina Veterinária e Zootecnia da USP. E.mail: cslucci@sti.com.br

3 Professor Associado da Faculdade de Zootecnia e Engenharia de Alimentos da USP. E.mail: rfranzol@usp.br
} 
PRESTON (1976), ocorre grande contribuição dos protozoários para a biomassa do rúmen, indicando que os protozoários não deixam o órgão em quantidades significantes para a nutrição do hospedeiro. A produtividade baixa de animais alimentados com dietas à base de cana-de-açúcar representaria uma forma de disfunção metabólica, afetando a ingestão de alimentos, associada ao aumento da população dos grandes protozoários holotricos.

$\mathrm{O} \mathrm{pH}$ do conteúdo ruminal é fator determinante na concentração e composição da populaçãode protozoários. Segundo HOBSON (1972), as espécies de Entodinium pareceram ser mais tolerantes às condições ácidas que os holotricos. Dietas à base de cana-de-açúcar apresentam $\mathrm{pH}$ alto e estável no rúmen, variando de 6,8 a 7,3, como resultado da alta taxa de fluxo salivar (LENG e PRESTON, 1976). Entretanto, há variabilidade individual no ambiente ruminal em animais sob o mesmo sistema de alimentação e outros fatores juntamente com o $\mathrm{pH}$ estão envolvidos na manutenção da população de protozários ciliados no rúmen (FRANZOLIN e DEHORITY, 1996a; FRANZOLIN e DEHORITY, 1996b).

Este trabalho teve por objetivo determinar as concentrações dos principais gêneros de protozoários, Entodinium, subfamília Diplodiniinae, Epidinium, Isotricha e Dasytricha, no conteúdo ruminal de ovinos, ao se substituir, em níveis crescentes, a silagem de milho por cana-de-açúcar na ração.

\section{Material e Métodos}

No presente experimento, foram utilizados oito ovinos machos, da raça Ideal, fistulados no rúmen, alojados em gaiolas de metabolismo e alimentados com dietas constituídas de silagem de milho, cana-deaçúcar e farelo de soja, além de $10 \mathrm{~g}$ de uma mistura mineral/animal/dia.

Quatro rações foram formuladas substituindo-se a silagem de milho (SM) pela cana-de-açúcar (CA) em níveis crescentes: A) $100 \%$ de SM; B) $66 \%$ de $\mathrm{SM}$ e $33 \%$ de $\mathrm{CA}$; C) $33 \%$ de $\mathrm{SM}$ e $66 \%$ de CA; e D) $100 \%$ de CA. As quantidades fornecidas de volumosos e concentrados foram calculadas de acordo com as necessidade protéicas e energéticas dos animais (NATIONAL RESEARCH COUNCIL - NRC, 1975). As rações foram fornecidas em porções iguais, quatro vezes ao dia, às 7, 12, 17 e $22 \mathrm{~h}$, e os tratamentos foram ajustados para o mesmo teor de proteína. Inicialmente fornecia-se o concentrado e, após seu total consumo, colocava-se o volumoso. Quando ocorriam sobras, estas eram desidratadas, moídas e retornadas ao seus respectivos animais no dia seguinte.

O delineamento estatísitico foi o quadrado latino $4 \times 4$ (PIMENTEL GOMES, 1985), com quatro sequências de tratamentos e duas repetições por sequência, em quatro subperíodos experimentais de 35 dias cada, sendo utilizados os dados obtidos de duas amostragens de líquido ruminal por subperíodo em cada animal. A análise de variância compreendeu o desdobramento dos graus de liberdade dos tratamentos para regressões linear e quadrática e desvios.

Amostras do conteúdo ruminal dos ovinos foram coletadas antes da primeira alimentação, via fístula, com o auxílio de uma pipeta com abertura larga, no $28^{\circ}$ e $35^{\circ}$ dia de cada subperíodo. Aproximadamente $10 \mathrm{~mL}$ de amostra foram preservados em igual volume de solução de formoldeído para posterior coloração e identificação de protozoários ciliados. As contagens dos protozoários foram realizadas conforme técnica descrita por DEHORITY (1993).

A taxa de passagem do líquido ruminal e o volume do rúmen foram determinados, em cada subperíodo, com a adição de $10 \mathrm{~g}$ de polietilenoglicol dissolvido em $50 \mathrm{~mL}$ de água e análise da concentração do marcador em uma e 24 horas após sua infusão, por colorimetria (HYDEN, 1956).

\section{Resultados e Discussão}

Constam da Tabela 1 as composições químicas dos alimentos utilizados e das rações experimentais. Observa-se que o equilíbrio nos teores de proteína bruta de todos os tratamentos foi alcançado, podendo ser melhorado apenas no tratamento $\mathrm{D}$, embora o resultado não tenha sido discrepante.

Os consumos médios de matéria seca em gramas por dia e em gramas por $\mathrm{kg}^{0,75}$ foram: 724,1 e 48,5; 701,2 e 46,1; 712,0 e 48,3; e 585,0 e 45,3, respectivamente para os tratamentos com ausência de cana-deaçúcar (CA), $33 \%$ de CA, $66 \%$ de CA e $100 \%$ de CA. Não houve diferenças significativas entre os tratamentos, mas observou-se diminuição no consumo médio, à medida que maiores quantidades de canade-açúcar foram empregadas.

Na Tabela 2, estão apresentados os resultados médios do número de protozoários, Entodium, Diplodiniinae, Epidinium, Dasytricha e Isotricha (por $\mathrm{mL}$ de líquido ruminal), e entre parênteses, a composição da fauna (\%). Observaram-se altos coeficientes de variação médios, entretanto, devido à dinâmica da população microbiana no rúmen, coefici- 
1454 Rev. bras. zootec.

Tabela 1 - Composição química bromatológica dos alimentos utilizados e das rações experimentais (base seca)

Table 1 - Chemical composition of the used feedstuffs and of the experimental diets (dry matter basis)

\begin{tabular}{|c|c|c|c|c|c|}
\hline $\begin{array}{l}\text { Alimentos } \\
\text { Feedstuffs }\end{array}$ & $\begin{array}{l}\text { MS } \\
D M\end{array}$ & $\begin{array}{l}\mathrm{PB} \\
C P\end{array}$ & $\begin{array}{l}\mathrm{EE} \\
E E\end{array}$ & $\begin{array}{l}\mathrm{ENN} \\
N N E\end{array}$ & $\begin{array}{c}\text { Cinzas } \\
\text { Ash }\end{array}$ \\
\hline $\begin{array}{l}\text { Cana-de-açúcar } \\
\text { Sugar cane }\end{array}$ & 31,90 & 2,62 & 0,52 & 69,49 & 1,13 \\
\hline $\begin{array}{l}\text { Silagem de milho } \\
\text { Corn silage }\end{array}$ & 22,60 & 7,00 & 1,47 & 61,60 & 3,53 \\
\hline $\begin{array}{l}\text { Farelo de soja } \\
\text { Soybean meal }\end{array}$ & 93,01 & 47,88 & 0,53 & 25,50 & 6,71 \\
\hline $\begin{array}{l}\text { Tratamentos } \\
\text { Treatments }\end{array}$ & & & & & \\
\hline $\begin{array}{l}\text { 100SM:0CA }{ }^{1} \\
\text { 67SM:33CA } \\
\text { 33SM:67CA } \\
\text { 0SM:100CA }\end{array}$ & $\begin{array}{l}26,47 \\
27,12 \\
28,56 \\
29,58\end{array}$ & $\begin{array}{r}10,33 \\
10,39 \\
10,36 \\
9,87\end{array}$ & $\begin{array}{l}2,63 \\
2,85 \\
2,12 \\
1,74\end{array}$ & $\begin{array}{l}54,09 \\
55,70 \\
56,72 \\
58,23\end{array}$ & $\begin{array}{l}6,85 \\
6,75 \\
6,67 \\
6,93\end{array}$ \\
\hline
\end{tabular}

${ }^{1} \mathrm{SM}=$ Silagem de milho (corn silage); CA = Cana-de-açúcar (sugar cane).

Tabela 2 - Concentração média e composição (\% entre parêntese) de total de protozoários e dos Entodinium, Diplodiniinae, Epidinium, Dasytrica e Isotricha (por $\mathrm{mL}$ de líquido ruminal) em ovinos alimentados com níveis crescentes de cana-deacúcar, em substituição à silagem de milho

Table 2 - Average concentrations and composition (\% in parenthesis) of the total protozoa, Entodinium, Diplodiniinae, Epidinium, Dasytrica e Isotricha (per $\mathrm{mL}$ of ruminal liquid) in sheep fed increasing levels of sugar cane in substitution of corn silage

\begin{tabular}{lccccc}
\hline \multicolumn{5}{c}{$\begin{array}{c}\text { Tratamentos } \\
\text { Treatments }\end{array}$} & \\
\cline { 2 - 5 } & 100SM:0CA $^{1}$ & 67SM:33CA & 33SM:67CA & 0SM:100CA & CV $^{2}(\%)$ \\
\hline Total & 53.005 & 41.416 & 39.421 & 22.744 & 28,5 \\
Entodinium & $46.372(87,5)$ & $36.169(87,3)$ & $36.723(93,2)$ & $21.341(93,8)$ & 29,1 \\
Diplodiniinae & $4.822(9,1)$ & $3.460(8,4)$ & $1.872(4,7)$ & $619(2,7)$ & 51,5 \\
Epidinium & $144(0,3)$ & $67(0,2)$ & 19 & 21 & 56,6 \\
Dasytricha & $1617(3,0)$ & $1716(4,1)$ & $807(2,0)$ & $754(3,3)$ & 38,4 \\
Isotricha & $50(0,1)$ & 4 & 0 & 9 & 116,8 \\
\hline
\end{tabular}

${ }^{1} \mathrm{SM}=$ Silagem de milho (corn silage); CA = Cana-de-açúcar (sugar cane).

$2 \mathrm{CV}=$ Coeficiente de variação (Coefficient of variation).

entes de variação elevados têm sido observados em experimentos dessa natureza (COLEMAN, 1979; MOURA MARINHO, 1982; e VEIRA, 1986).

Houve diminuição linear $(\mathrm{P}<0,01)$ na concentração total dos protozoários ciliados por $\mathrm{mL}$ de líquido ruminal, com o aumento nos níveis de substituição da silagem de milho pela cana-de-açúcar nas rações ( $\mathrm{Y}=223,41-0,719 \mathrm{X}$, em que $\mathrm{Y}$ é o número de protozoários e $\mathrm{X}$, a porcentagem de cana-de-açúcar na ração). Esse mesmo efeito também foi observado $(\mathrm{P}<0,02)$ com os ciliados pertencentes ao gênero Entodinium ( $\mathrm{Y}=208,33$ - 0,620 X).

As concentrações médias de ciliados encontradas foram inferiores às observadas por BIRD et al. (1979), fornecendo dietas à base de cana-de-açúcar para ovinos (5,0 × $10 \% / \mathrm{mL}$ de líquido ruminal). O nível de energia na dieta pode ter influenciado esses valores baixos, uma vez que MICHALOWSKI (1975), LYLE et al. (1981) e DENNIS et al. (1983),encontraram 1,$5 ; 2,5$; e 4,1 x $10^{5}$ protozoários por $\mathrm{mL}$ de líquido ruminal em dietas com alta, média e baixa energia, respectivamente, em que a energia foi aumentada com maior uso de concentrados.

$\mathrm{O}$ gênero Entodinium predominou entre os protozoários para todas as dietas estudadas, que variou de 87,3 a $97,8 \%$ da concentração total, con- 
cordando com a citação de DEHORITY (1991) de que, em geral, cerca de $90 \%$ da fauna de ruminantes é formada de entodínios. No tratamento com canade-açúcar como único volumoso, o seu número foi de $2,0 \times 10^{4}$ por $\mathrm{mL}$, sendo inferior às concentrações encontradas por MINOR et al. (1977), de 3,0 x 105/mL, LENG et al. (1981), de 4,0 x 105/mL, e VALDEZ et al. (1977), de 1,0 x 105\% $\mathrm{mL}$. Esses autores também observaram predominância dos Entodinium, mas citaram que os Isotricha e Dasytrica (holotrichos) sobrepujam em até 100 vezes o tamanho dos Entodinium. A substituição total da silagem de milho por cana-de-açúcar aumentou a porcentagem de Entodinium de 87,5 para $93,8 \%$, porém diminuiu a de Diplodiniinae de 9,1 para 2,7\%, mantendo-se a mesma proporção de Dasytricha (média 3,2\%).

MINOR et al. (1977) não detectaram diferença entre a biomassa de protozoários de animais alimentados com cana-de-açúcar ou polidura de arroz. Segundo CHURCH (1974), os holotricos aparecem em maior número nos animais alimentados com açúcares solúveis. No presente trabalho, o número de Dasytrica foi bem maior que de Isotrica, contrariamente ao registrado nos experimentos de MINOR et al. (1977) e VALDEZ et al. (1977). Segundo esses autores e, ainda, LENG e PRESTON (1976), as dietas com cana-de-açúcar indicam população de protozoários dividida entre holotrica (Isotricha e Dasytricha) e Entodinium, mas, em termos de biomassa, os holotrichos prevalecem, com os Isotricha sobrepujando os Dasytricha, fenômeno não ocorrido neste trabalho. O grande tamanho de protozoários pode afetar seus fluxos no rúmen (LENG, 1982), pois as espécies menores, provavelmente escapam da fermentação ruminal. Isto implica no baixo aproveitamento de proteína microbiana na forma de protozoários pelo hospedeiro, devido ao menor fluxo para as porções inferiores do trato gastrintenial, podendo ser uma explicação para a baixa produtividade dos animais alimentados com cana-de-açúcar (COLEMAN, 1979). Os holotricos utilizam açúcares solúveis para seu metabolismo e bovinos alimentados com cana-de-açúcar tendem a ter uma população de protozoários no rúmen com predominância dos holotricos (RYLE e ØRSKOV, 1987).

Na Tabela 3, são observados os resultados médios e os coeficientes de variação do volume do líquido ruminal, em litros, a taxa de passagem do líquido ruminal por hora e por dia e os valores de pH. Não foram observadas diferenças significativas entre os tratamentos em nenhum desses parâmetros avaliados $(\mathrm{P}>0,05)$.

Embora vários fatores possam influir no volume do conteúdo ruminal, PRIEGO e LORA (1978) e ROBLES et al. (1981) verificaram pouca variação no volume ruminal de ovinos que consumiam diversos tipos de forragens. NOGUEIRA FILHO et al. (1977) e BIONDI et al. (1978), ao substituirem silagem de milho por cana-de-açúcar, observaram diminuição gradativa do consumo de MS, o que pode ser explicado pela baixa degradabilidade ruminal da fibra da cana-de-açúcar (PATE, 1981), fazendo este alimento ter maior tempo de

Tabela 3 - Valores médios de volume do rúmen (litros), taxa de passagem do líquido ruminal por hora e $\mathrm{pH}$ euminal em ovinos alimentados com níveis crescentes de cana-de-açúcar em substituição à silagem de milho

Table 3 - Average values of rumen volume (liters), passage rate per hour and $\mathrm{pH}$ in sheep fed increasing levels of sugar cane in substitution of corn silage

\begin{tabular}{|c|c|c|c|c|c|}
\hline & \multicolumn{4}{|c|}{$\begin{array}{l}\text { Tratamentos } \\
\text { Treatments }\end{array}$} & \multirow[b]{2}{*}{$\mathrm{CV}^{2}(\%)$} \\
\hline & $\begin{array}{l}\text { 100SM: } \\
\text { OCA }\end{array}$ & $\begin{array}{l}\text { 67SM: } \\
33 C A\end{array}$ & $\begin{array}{l}\text { 33SM: } \\
67 C A\end{array}$ & $\begin{array}{l}\text { 0SM: } \\
100 C A\end{array}$ & \\
\hline $\begin{array}{l}\text { Volume ruminal (litros) } \\
\text { Rumen volume }\end{array}$ & 4,8 & 5,7 & 5,4 & 5,2 & 21,9 \\
\hline $\begin{array}{l}\text { Taxa de passagem (por hora) } \\
\text { Passage rate }\end{array}$ & 0,11 & 0,11 & 0,11 & 0,11 & 17,9 \\
\hline $\begin{array}{l}\text { Taxa de passagem (por dia) } \\
\text { Passage rate }\end{array}$ & 2,64 & 2,64 & 2,64 & 2,64 & 17,9 \\
\hline $\mathrm{pH}$ & 6,7 & 6,6 & 6,8 & 6,9 & 1,49 \\
\hline
\end{tabular}


1456 Rev. bras. zootec.

permanência no rúmen, o que poderia, teoricamente, ser compensado em parte pelo aumento do turnover da fase líquida (HUNGATE, 1966).

VARGA e PRIGGE (1982) encontraram volumes ruminais entre 5,7 e 6,4 litros para dietas com feno de alfafa e taxa de passagem de 0,071 a $0,073 / \mathrm{h}$, enquanto, neste experimento, os volumes ruminais foram levemente inferiores, ficando entre 4,8 e 5,7 1itros, e as taxas de passagem foram superiores $(0,109$ a $0,110 / \mathrm{h})$. Já DEHORITY e PURSER (1970) e MUDGAL et al. (1982) encontraram volumes de rúmen de ovinos de 3,5 e de 6,1 a 7,4 litros, respectivamente, com taxas de passagem de 1,87 a 2,88 por dia, próximas às observadas no presente trabalho.

$\mathrm{O} \mathrm{pH}$ do rúmen aumentou linearmente com o incremento de cana-de-açúcar nas rações $(\mathrm{P}<0,05)$ $(\mathrm{Y}=6,631+0,0015 \mathrm{x}$, em que y é o $\mathrm{pH}$ do rúmen e $x$, a porcentagem de cana). Valores semelhantes de $\mathrm{pH}$ ruminal foram encontrados em animais alimentados à base de cana-de-açúcar por VALDEZ et al. (1977), FERREIRO et al. (1978) e FERNANDEZ e GILL (1980). O pH elevado seria resultante de insalivação mais abundante nas dietas com cana-de-açúcar, em virtude de maior tempo despendido com a mastigação e ruminação das rações. Os resultados obtidos no presente experimento diferem dos obtidos por LENG e PRESTON (1976), os quais, ao utilizarem dietas contendo grandes quantidades em amido, constataram que, em animais com valores baixos de $\mathrm{pH}$ no rúmen, os protozoários geralmente estão ausentes; ao inverso, com níveis altos de cana-de-açúcar, boas populações de protozoários são sempre obtidas.

\section{Conclusões}

A substituição de silagem de milho por cana-deaçúcar provoca diminuição linear no número dos protozoários e de Entodinium no conteúdo ruminal.

A substituição de silagem de milho por canade-açúcar promove aumento linear no $\mathrm{pH}$ do conteúdo ruminal.

O gênero Entodinium predomina entre os protozoários na fauna ruminal em ovinos alimentados à base de cana-de-açúcar, variando de 87,3 a 97,8\% da concentração total.

\section{Agradecimento}

À Prof. ${ }^{a}$ Dr ${ }^{a}$ Catarina Abdalla Gomide da FZEA/ USP, pelo apoio prestado durante a execução prática na última fase experimental.

\section{Referências Bibliográficas}

BIONDI, P., CAIELLI, E.L., FREITAS, E.A.N. et al. 1978. Substituição parcial e total da silagem de milho por cana-deaçúcar como únicos volumosos para vacas em lactação. Bol. Ind. Anim., 35(1):45-55.

BIRD, S.H., HILL, M.K., LENG, R.A. 1979. The effects of defaunation on the rumen on growth of lambs on low-proteinhigh-energy diets. Brit. J. Nut., 42(1):81-87.

CHRISTIANSEN, W.C., WOODS, W., BURROUGHS, W. 1964. Ration characteristics influencing rumen protozoal population. J. Anim. Sci., 23(4):984-988.

CHURCH, D.C. 1974. Fisiologia digestiva y nutricion de las rumiantes. vol 1. Zaragoza: Editorial Acribia. 483p.

COLEMAN, G.S. 1979. The role of rumen protozoa in the metabolism of ruminants given tropical feeds. Trop. Anim. Prod., 4:199-213.

DEHORITY, B.A. 1991. Rumen microbiology. Wooster, USA: OARDC/OSU. 87p.

DEHORITY, B.A. 1993. Laboratory manual for classification and morphology of rumen ciliate protozoa. Florida: CRC Press Inc. 96p.

DEHORITY, B.A, PURSER, D.B. 1970. Factors affecting the establishment and numbers of holotrich protozoa in the ovine rumen. J. Anim. Sci., 30(3):445-450.

DENNIS, S.M., ARAMBEL, M.J., BARTLEY, E.E. et al. 1983. Effect of energy concentration and source of nitrogen on numbers and types of rumen protozoa. J. Dairy Sci., 66(6): $1248-1254$

FERNANDEZ, A., GILL, M. 1980. La poblacion de protozoários en el rumen de novillos recebiendo dietas basadas en caña integral, caña descortezada ou melaza y forraje. Trop. Anim. Prod., 5(1):84-85.

FERREIRO, H.M., SUTHERLAND, T.M., WILSON, A. et al. 1978. Fattening cattle with sugar cane: a comparison of different supplements. Trop. Anim. Prod., 2:309-314.

FRANZOLIN, R., DEHORITY, B.A. 1996a. Effect of prolonged high-concentrate feeding on ruminal protozoa concentrations. J. Anim. Sci., 74(11):2803-2809.

FRANZOLIN, R., DEHORITY, B.A. 1996b. Efeitos do pH ruminal e ingestão alimentar na defaunação em ovinos sob rações concentradas. R. Soc. Bras. Zootec., 25(6):1207-1215.

HOBSON, P.N. 1972. Physiological characterístics of rumen microbes and relation to diet and fermentation patterns. Proc. Nutr. Soc., 31(2):135-139.

HUNGATE, R.E. 1966. The rumen and its microbes. New York. Academic Press Inc. 533p.

HYDEN, S. 1956. A turbidimetric method for the determination of higher polyethyleneglycol in biological material. $\mathrm{Kgl}$. Lantbruks-Hogskol. Ann. Uppsala, 22:139-145.

LENG, R.A. 1982. Dynamics of protozoa in the rumen of sheep. Brit. J. Nutr., 48(2):399-415.

LENG, R.A., PRESTON, T.R. 1976. Sugar cane for cattle production: present constraints, perspectives and research priorities. Trop. Anim. Prod., 1(1):1-22.

LENG, R.A., GILL, M., KEMPTON, T.J. et al. 1981. Kinetics of large ciliate protozoa in the rumen of cattle given sugar cane diets. Brit. J. Nutr., 46(2):371-384.

LYLE, R.R., JOHNSON, R,R., WILHITE, J.V. et al. 1981 Ruminal characteristics in steers as affected by adaptation from forage to all concentrate diets. J. Anim. Sci., 53(5):1383-1390.

MICHALOWSKI, T. 1975. The effect of certain feedingstuffs on rumen ciliate protozoa in vitro. J. Agric. Sci., 85(1):151-158. 
MINOR, S., MAcLEOD, N.A., PRESTON, T.R. et al. 1977. Studies on digestion in different sections of the intestinal tract. of bulls fed sugar cane/urea with different supplements. Trop. Anim. Prod., 2(1):163-174.

MOURA MARINHO, A.A. de. 1982. Ciliados do rúmen - sua dinâmica e importância no metabolismo digestivo dos ruminantes. revisão. Rev. Port. Cienc. Veter., 77(464):241-259.

MUDGAL, V.D., DIXON, R.M., KENNEDY, P.M. et al. 1982. Liquid, partide and microbial markers in the rumen of sheep. J. Anim. Sci., 54(5):1051-1055.

NATIONAL RESEARCH COUNCIL - NRC. 1975. Nutrient requirements of sheep. Washington: National Academy of Sciences. 72p.

NOGUEIRA FILHO, J.C.M., LUCCI, C.S., ROCHA, G.L. et al. 1977. Substituição parcial da silagem de sorgo por cana-deaçúcar como únicos volumosos para vacas em lactação. Bol. Ind. Anim., 34(1):75-84.

PATE, F.M. 1981. Fresh chopped sugar cane in growing-finishing steer diets. J. Anim. Sci., 53(4):881-887.

PIMENTEL GOMES, F. 1985. Curso de estatística experimental. 3.ed., Piracicaba/SP: ESALQ. 467p.

PRIEGO, A., LORA, J.A. 1978. Effect of feeding frequency and supplementation with wheat bran on voluntary intake and rumen function of cattle fed sugar cane. Trop. Anim. Prod., $3: 211-217$.
ROBLES, A.Y., BELYEA, R.L., MARTZ, F.A. et al. 1981. Intake, digestibility, ruminal characteristics and rate of passage of orchardgrass diets fed to sheep. J. Anim. Sci., 53(2):489-493.

RYLE, M., ØRSKOV, E.R. 1987. Ciliados de la panza y piensos tropicales. Rev. Mundial Zootec., 64(10):21-30.

VALDEZ, R.E., ALVAREZ, F.J., FERREIRO, H.M. et al. 1977. Rumen function in cattle given sugar cane. Trop. Anim. Prod., 2:260-272.

VARGA, G.A., PRIGGE, E.C. 1982. Influence of forage species and level of intake on ruminal turnover rate. J. Anim. Sci., 55(6):1498-1504.

VEIRA, D.M. 1986. The role of ciliate protozoa in nutrition of the ruminant. J. Anim. Sci., 63(5):1547-1560.

Recebido em: 29/10/99

Aceito em: 04/04/00 